\title{
Two New Coumarins from the Leaves of Phellodendronamurense.
}

\author{
Zhao Zhang, Guangzhi Li, Lingchao Dai, Bengang Zhang and Jianyong Si*
}

Key Laboratory of Bioactive Substances and Resources Utilization of Chinese Herbal Medicine, Ministry of Education of Institute of Medicinal Plant Development, Chinese Academy of Medical Sciences, Peking Union Medical College, Beijing 100193, China

Received: February 26, 2017; Accepted: April 16, 2017; Published: May 4, 2017

*Corresponding author: Jianyong Si, Key Laboratory of Bioactive Substances and Resources Utilization of Chinese Herbal Medicine, Ministry of Education of Institute of Medicinal Plant Development, Chinese Academy of Medical Sciences, Peking Union Medical College, Beijing 100193, China; Telephone and fax numbers: +86010-57833299; E-mail: Jianyongsi@126.com

\begin{abstract}
Two new coumarins, PhellodenolsI (1) and J (2), were isolated from the leaves of Phellodendronamurense. The structures of these compounds were elucidated by extensive spectroscopic analysis. The two new compounds were tested against Hela, AGS, and MGC-803 human cancer cell lines and exhibited cytotoxic activities with IC50 values between $18.71 \pm 5.23 \mu \mathrm{M}$ and $36.92 \pm 4.72 \mu \mathrm{M}$.
\end{abstract}

Key words: Phellodendronamurense; Leaves; Coumarins; Cytotoxic activities

\section{Introduction}

Phellodendronamurense Rupr is an important member of the genus Phellodendron belonging to the family Rutaceae [1]. It mainly distributes in Japan, Korea, and northeast of China, and has been used as a kind of traditional medicine for the treatment of intestinal function controlling and stomach aches for years [2]. Modern pharmacological studies have established the anti-inflammatory, anti-psychic, and antioxidant activities of this plant [3]. Previous phytochemical studies reveal the presence of coumarins, alkaloids, limonoidal triterpenes as well as phytosterols and their fatty acid estersin this plant [4]. In our study, two new coumarins, phellodenolsI (1) and J (2), were isolated from the leaves of P.amurense (Figure 1). In this paper, we describe the structure elucidation of the two new compounds and their cytotoxicity against any human cancer cell lines.

Graphic abstract
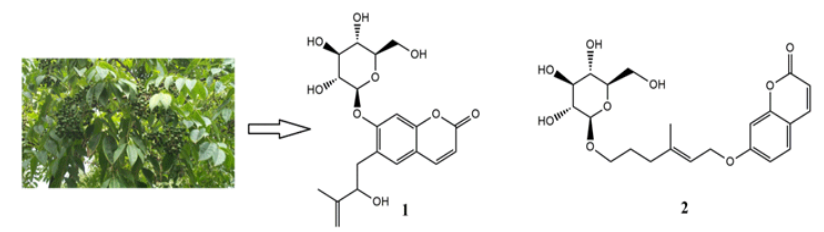

(a)

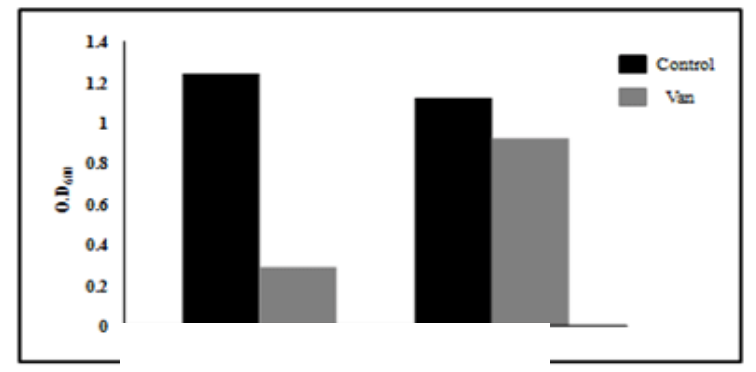

Figure 1: The structures of 1 and 2

\section{Materials and methods}

\section{General experimental procedure}

Optical rotations were determined using a Perkin-Elmer 341 digital polarimeter. IR spectra were recorded on a FTIR8400 S spectrum and UV spectra were obtained on a Shimadzu UV2550 spectrometer. Melting points were determined without correction on a Fisher-Johns melting point apparatus (FisherJohns Scientific Company). High resolution mass spectra were measured on a Thermo Scientific LTQ-Obitrap XL (Thermo Scientific, Bremen, Germany). The NMR spectra were performed on Bruker AV Ш 600 spectrometers with TMS as an internal standard. Column chromatography was performed on silica gel (100-200 mesh, Qingdao Haiyang Chemical Co., Ltd., Qingdao, P. R. China) and Sephadex LH-20 (Pharmacia Biotech, Sweden). Precoated silica gel plates (F254, 0.25 mm, Qingdao Haiyang Chemical Co., Ltd., Qingdao, P. R. China) were used for TLC analysis. All solvents used for HPLC were HPLC grade (Fisher Scientific).

\section{Plant material}

The leaves of P. amurense were collected in November 2008 from Beijing, China and authenticated by ZZhang. A voucher specimen of the plant (DLC-20101108) has been deposited at the herbarium of Institute of Medicinal Plant Development, Beijing, China. 


\section{Extraction and isolation}

The shade-dried leaves of P. amurense $(5.0 \mathrm{~kg})$ were crushed and refluxed with $95 \%$ EtOH $(2 \times 20 \mathrm{~L})$ two times for $3 \mathrm{~h}$. The resulting solutions were evaporated under reduced pressure to yield a dark brown residue (760 g), which was suspended in water and extracted by chloroform(1:1, v/v). The water-soluble portion (152 g) was fractionated into four fractions (Fr. A D) by Diaion HP-20 chromatography eluting with water-ethanol (1:0 to 1:0, v/v). Fr. B (22 g) was crude separated on silica gel chromatography using the mobile phase of $\mathrm{CHCl} 3-\mathrm{MeOH}$ (10:1 to $0: 1, v / v$ ) to obtain twenty fractions (Fr. B1 20). Fr. B16 (3.0 g) was further separated over a Sephadex LH-20 column eluting with $\mathrm{MeOH}$, yielding compound 1 (2.7mg). Fr. C (32 g) was subjected to chromatography on a silica gel column eluting with $\mathrm{CHCl} 3-\mathrm{MeOH}$ (20:1 to $0: 1, v / v)$, to obtain ten fractions (Fr. C1 10). Fr.C3 (0.8 g) was subjected to preparative thin layer chromatography (PTLC) eluting with $\mathrm{CHCl} 3-\mathrm{MeOH}-\mathrm{H} 2 \mathrm{O}$ (17:3:2). Finally, compound 2 was obtained (2.3 mg).

6-(2'-hydroxy-3'-methylbut-3'-en-1'-yl)-7-(2",3",4"'-trihydroxy6"'-(hydroxymethyl) te trahyd ro-2 H-pyran-1"'-yl) oxy)-2 Hchromen-2-one (1): white powder $(\mathrm{MeOH}) ; \mathrm{mp} 162-163{ }^{\circ} \mathrm{C}$; IR $v \max 3430,1726,1622,1506 \mathrm{~cm}-1$; UV (MeOH) $\lambda \max (\log \varepsilon) 324$ (4.05), 274(3.74) nm; HRESIMS [M+Cl]- :443.1112 (calcd for C20H24ClO9, 443.1109); $[\alpha] 20 \mathrm{D}=-25.9$ (c 0.17, MeOH); $1 \mathrm{H}$ and 13C NMR see Table 1.

$7-\{[(E)-3$ '- methyl-6' - [(2"',3", 4"'-trihydroxy-6"'(hydroxymethyl)tetrahydro-2H-pyran-1"'-yl)oxy] hex-2'-en-1'-yl] oxy\}-2H-chromen-2-one (2): white powder (MeOH); mp 90$91{ }^{\circ} \mathrm{C}$; IR vmax 3354, 1727, 1612,1507 cm-1; UV (MeOH) $\lambda \max$ (log ع) 323 (4.01) nm; HRESIMS [M+Na]+ 459.1615(calcdfor C22H28NaO9, 459.1631); $[\alpha] 20 \mathrm{D}=-69.0($ c 0.13, MeOH); $1 \mathrm{H}$ and13C NMR see Table 1.

Table 1: ${ }^{1} \mathrm{H}$ and ${ }^{13} \mathrm{C}$ NMR (600MHz) data for 1 and 2 in DMSO- $d_{6}$

\begin{tabular}{|c|c|c|c|c|}
\hline & \multicolumn{2}{|r|}{1} & \multicolumn{2}{|c|}{2} \\
\hline & $\delta_{\mathrm{c}}$ & $\delta_{\mathrm{H}}(J, \mathrm{~Hz})$ & $\delta_{\mathrm{c}}$ & $\delta_{\mathrm{H}}(J, \mathrm{~Hz})$ \\
\hline 1 & & & & \\
\hline 2 & 160.3 & & 160.3 & \\
\hline 3 & 112.8 & $6.29(\mathrm{~d}, 9.0)$ & 112.3 & $6.28(d, 9.6)$ \\
\hline 4 & 144.3 & $7.97(\mathrm{~d}, 9.0)$ & 144.5 & $7.97(d, 9.6)$ \\
\hline 5 & 130.1 & $7.48(\mathrm{~s})$ & 129.4 & $7.61(\mathrm{~d}, 8.4)$ \\
\hline 6 & 125.3 & & 112.8 & $6.95(\mathrm{dd}, 8.4,2.4)$ \\
\hline 7 & 158.3 & & 161.6 & \\
\hline 8 & 101.7 & $7.09(\mathrm{~s})$ & 101.3 & $6.99(\mathrm{~d}, 2.4)$ \\
\hline $4 a$ & 112.4 & & 112.2 & \\
\hline $8 a$ & 153.6 & & 155.3 & \\
\hline $1^{\prime}$ & 35.9 & $\begin{array}{l}2.87(\mathrm{dd}, 8.4,13.8) \\
2.59(\mathrm{dd}, 8.4,13.8)\end{array}$ & 65.1 & $4.65(d, 6.6)$ \\
\hline $2^{\prime}$ & 72.7 & $4.27(\mathrm{~m})$ & 118.8 & $5.46(t, 6.6)$ \\
\hline $3^{\prime}$ & 148.3 & & 141.1 & \\
\hline $4^{\prime}$ & 109.7 & $\begin{array}{l}4.87(\mathrm{~s}) \\
4.69(\mathrm{~s})\end{array}$ & 35.1 & $2.10(\mathrm{~m})$ \\
\hline $5^{\prime}$ & 17.9 & $1.75(3 \mathrm{H}, \mathrm{s})$ & 27.2 & $1.65(\mathrm{~m})$ \\
\hline $6^{\prime}$ & & & 68.0 & $3.74^{a}$ \\
\hline $7^{\prime}$ & & & 16.4 & $1.72(3 \mathrm{H}, \mathrm{s})$ \\
\hline $1^{\prime \prime}$ & 100.1 & $5.01(\mathrm{~d}, 7.8)$ & 102.9 & $4.09(\mathrm{~d}, 7.8)$ \\
\hline $2^{\prime \prime}$ & 73.3 & $3.29 \sim 3.34^{a}$ & 73.4 & $2.95^{a}$ \\
\hline $3^{\prime \prime}$ & 76.8 & $3.30 \sim 3.33(\mathrm{~m})$ & 76.7 & $3.04^{a}$ \\
\hline $4^{\prime \prime}$ & 69.7 & $3.17^{a}$ & 70.0 & $3.05^{a}$ \\
\hline $5^{\prime \prime}$ & 77.1 & $3.43 \sim 3.47(\mathrm{~m})$ & 76.7 & $3.13^{a}$ \\
\hline $6^{\prime \prime}$ & 60.7 & $3.73(\mathrm{dd}, 11.8,6.6)$ & 61.0 & $3.65(\mathrm{dd}, 11.8,6.6)$ \\
\hline
\end{tabular}




\section{Cytotoxicity assay}

Cytotoxic testing was performed as the following previously described protocols $[5,6]$.The cytotoxicity of the two new compounds were assessed using the MTT method against AGS, MGC-803 and Hela human cancer cell lines. AGS cells were cultured in Ham's F12 medium supplemented with 10\% fetal bovine serum, while MGC-803 and Hela cells were cultured in DMEM medium. Cells were cultured in a 96-well plate and cultured $24 \mathrm{~h}$. Dimethyl sulfoxide (DMSO) was used to dissolve the tested compounds and Taxol was used as a positive control Each concentration was tested in triplicate. After incubation at $37{ }^{\circ} \mathrm{C}$ in $5 \%$ CO2 for $48 \mathrm{~h}, 10 \mu \mathrm{L}$ of MTT ( $4 \mathrm{mg} / \mathrm{mL}$ ) was added to each well and cultured for another $4 \mathrm{~h}$. And then the supernatant was discarded and $150 \mu \mathrm{L}$ DMSO was added to each well. The absorbance was detected on a microplate reader at a wavelength of $570 \mathrm{~nm}$.

\section{Results and Discussion}

After successive stages of chromatography, the leaves of P. amurense extract yielded two new coumarins, phellodenolsI (1) and J (2). Their structures were elucidated on the basis of detailed analysis of the spectroscopic data.

Compound 1 was obtained as a white powder from in $\mathrm{MeOH}$. Its molecular formula was deduced as $\mathrm{C} 20 \mathrm{H} 2409$ on the basis of 13CNMR data and the $[\mathrm{M}+\mathrm{Cl}]$ - ion peak at $\mathrm{m} / \mathrm{z} 443.1112$ (calcd for C20H24CI09, 443.1109) in the HRESIMS. The IR absorption bands indicated the presence of hydroxyl groups (3430 cm-1), a carbonyl group (1726 cm-1) and a double bond (1622 cm-1). The UV spectrum exhibited an absorption maximum for coumarin moiety $(324 \mathrm{~nm})$ [5]. The planar structure of 1 was elucidated by analysis of the NMR spectra data. The 13CNMR and heteronuclear singlequantum coherence (HSQC) spectra displayed 20 carbon signals. The downfield resonances $(\delta \mathrm{C} 109.7,148.3$ ) in the 13Cspectrum showed the presence of a terminal double bond group. Other carbon signals were a methyl group, one primary oxygenated carbon, one aliphatic methylene carbon, and five oxygenated methine carbons. The nine remaining downfield resonances ( $\delta \mathrm{C} 101.7,112.4,112.8,125.3,130.1,144.3,153.6,158.3,160.3$ ) were reminiscent of a substituted 7-hydroxycoumarin, and the presence of it was confirmed by resonances at $\delta \mathrm{H6} 6.29$ (d, 9.0 $\mathrm{Hz}$ ), 7.09 (s), 7.48 (s), 7.97 (d, $9.0 \mathrm{~Hz}$ ) in the 1H NMR spectrum. Besides, the characteristic resonances $(\delta \mathrm{C}: 60.7,69.7,73.3,76.8$, $77.1,100.1 ; \delta \mathrm{H}: 3.17 \sim 3.73,5.01)$ indicated the presence of a sugar moiety, while the other five signals indicated an isoprenoid residue. The $1 \mathrm{H}-1 \mathrm{H}$ COSY spectrum showed a spin system signals $\left(\mathrm{H}-1^{\prime} / \mathrm{H}-2^{\prime}\right)$, which suggested the presence of the partial structure $\left(\mathrm{C}-1^{\prime}-\mathrm{C}-2^{\prime}\right)$ as shown in Figure 2. In the HMBC spectrum (Figure $2)$, correlations were observed for $\mathrm{H}-2^{\prime}(\delta \mathrm{H} 4.27)$ to $\mathrm{C}^{-} 5^{\prime}(\delta \mathrm{C} 17.9)$, C-4' ( $\delta \mathrm{C} 109.7) ; \mathrm{H}-4^{\prime}(\delta \mathrm{H} 4.69)$ to $\mathrm{C}-5^{\prime}(\delta \mathrm{C} 17.9), \mathrm{C}-2^{\prime}(\delta \mathrm{C} 72.7)$ and $\mathrm{H}-1^{\prime}(\delta \mathrm{H} 2.87)$ to $\mathrm{C}^{\prime}(\delta \mathrm{C} 148.3)$, suggesting the presence of the 2'-hydroxy-3'-methylbut-3'-ene moiety that was positioned at C-6 by the correlations of $\mathrm{H}-1^{\prime}(\delta \mathrm{H} 2.87)$ to $\mathrm{C}-5(\delta \mathrm{C} 130.1)$ and $\mathrm{C}-7(\delta \mathrm{C}$ 158.3). Besides, the correlations from $\mathrm{H}-1^{\prime \prime}$ to $\mathrm{C}-7$ ( $\delta \mathrm{C}$ 158.3) established the glucosidic bond at C-7. Since the NMR data of the sugar moiety was similar to that of the published spectra of $\beta$-D- a)

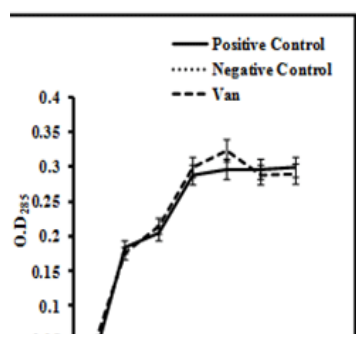

(b)

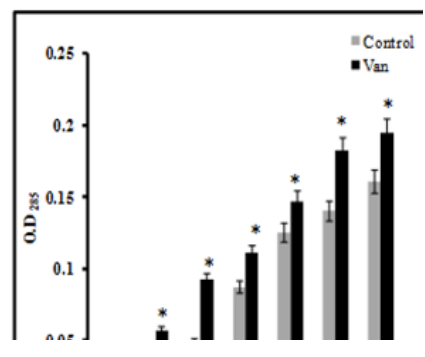

Figure 2: Key 2D NMR correlations of 1 and 2

glucopyranoside, as well as the large coupling constants between $\mathrm{H}-1^{\prime \prime}$ and $\mathrm{H}-2^{\prime \prime}(\mathrm{J}=7.8 \mathrm{~Hz})$, the sugar unit was established as $\beta$-D-glucopyranoside, finally $[7,8]$. Therefore, the structure of 1 was elucidated as 6-(2'-hydroxy-3'-methylbut-3'-en-1'-yl)-7(2",3", 4"'-trihydroxy-6"-(hydroxymethyl)tetrahydro-2H-pyran1 "'-yl)oxy)-2H-chromen-2-one. We named the new compound as phellodenoll.

Compound 2 was obtained as a white powder $(\mathrm{MeOH})$, and analyzed for $\mathrm{C} 22 \mathrm{H} 2809$ from HRESIMS, as a prominent peak was observed at m/z 459.1615, calcd for C22H28NaO9 459.1631. The UV spectrum showed an absorption maximum at $323 \mathrm{~nm}$, indicating 2 was also a kind of coumarin compound. The NMR data of 2 (Table 1) were similar to those of 1 , containing a coumarin moiety and a sugar moiety, except that the coumarin moiety of 2 was 7-hydroxycoumarin, as the ABX spin system signals [ $6 \mathrm{H} 7.61$ (d, J = 8.4 Hz, H-5); 6.95 (dd, J = 2.4, 8.4 Hz, H-6); 6.99 (d, J = $2.4 \mathrm{~Hz}$, $\mathrm{H}-8)]$ in the $1 \mathrm{HNMR}$ spectrum. Besides, the oxygenated tertiary carbon $\mathrm{C}-2^{\prime}(\delta \mathrm{c} 72.7)$ was replaced by two secondary carbons $(\delta c 65.1,68.0)$, and two more carbon signals showed in the 13CNMR spectrum of 2 . In the $1 \mathrm{H}-1 \mathrm{H}$ COSY spectrum, spin system signals $\left(\mathrm{H}-1^{\prime} / \mathrm{H}-2^{\prime} ; \mathrm{H}-4^{\prime} / \mathrm{H}-5^{\prime} / \mathrm{H}-6^{\prime}\right)$ suggested the presence of the

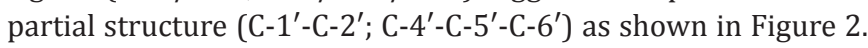
The HMBC correlations between H-1'/C-7, H-4'/C-2', H-7'/C-2', and $\mathrm{H}-\mathrm{7}^{\prime} / \mathrm{C}-\mathrm{C}^{\prime}$ showed the presence of 3-methylhex-2'-enoxy group located at C-7 of the coumarin moiety. The geometry about 2', 3'-trisubstituted double bound was determined, like pituranthoside, as trans rather than cis based on the fine splitting pattern of the 2'-vinyl proton $(\mathrm{t}, \mathrm{J}=6.6 \mathrm{~Hz})[9,10]$.Furthermore, the HMBC correlation between $\mathrm{H}-\mathrm{1}^{\prime \prime} / \mathrm{C}-\mathrm{6}^{\prime}$ and the similarity of NMR data of the sugar moiety of 2 with that of 1 suggested that C-6' was glycosylated with $\beta$-D-glucopyranose. Finally, the structure of 2 was determined as 7-(( (E)-3'-methyl-6'-((2", 3", 4"-trihydroxy-6"-(hydroxymethyl) tetrahy dro-2H-pyran-1"-yl) oxy) hex-2'-en-1'-yl) oxy)-2H-chromen-2-one, and we named this compound PhellodenolJ. Besides, a plausible biogenetic origin of this compound could be traced back to the monoterpenoid coumarins, pituranthoside, as shown in the supplementary data.

The new compounds 1 and 2 were evaluated for their cytotoxic activity against Hela, MGC-803, and AGS cell lines using the MTT assay. The compound 1 exhibited moderate cytotoxic activity against MGC-803 (IC50 $=28.53 \pm 6.03 \mu \mathrm{M}$ ), Hela $($ IC50 $=18.71 \pm 5.23 \mu \mathrm{M})$ and AGS $($ IC50 $=34.31 \pm 4.25 \mu \mathrm{M})$ cancer 
cell lines relative to that of the positive control (IC50 $=2.53 \pm 2.25$, $4.60 \pm 3.01$, and $4.52 \pm 3.04 \mu$ Magainst MGC-803, Hela, and AGS, respectively). The compound 2 also displayed cytotoxicity against MGC-803 (IC50=36.92 $\pm 4.72 \mu \mathrm{M})$, Hela $($ IC50 $=24.92 \pm 3.48 \mu \mathrm{M})$ and AGS (IC50 $=35.76 \pm 3.01 \mu \mathrm{M}$ ) cell lines (Table 2).Consideration the structures of 1 and 2 and the relevant reports suggested that the 7-0-substitution of the coumarin moiety may play a role in the cytotoxic activity of the new compounds [11].

Table 2: The cytotoxic activity of compounds 1 and 2.

\begin{tabular}{|c|c|c|c|}
\hline \multirow[t]{2}{*}{ Compound } & \multicolumn{3}{|c|}{$\mathrm{IC}_{50}(\mu \mathrm{M})^{a}$} \\
\hline & Hela & MGC-803 & AGS \\
\hline 1 & $18.71 \pm 5.23$ & $28.53 \pm 6.03$ & $34.31 \pm 4.25$ \\
\hline 2 & $24.92 \pm 3.48$ & $36.92 \pm 4.72$ & $35.76 \pm 3.01$ \\
\hline Taxol $^{b}$ & $4.60 \pm 3.01$ & $2.53 \pm 2.25$ & $4.52 \pm 3.04$ \\
\hline
\end{tabular}

\section{Acknowledgements}

This work was financially supported by the National Natural Science Foundation of China (81473305), special foundation for basic Research program of China (2015FY111500), and program for Innovative Research Team in IMPLAD.

\section{References}

1 Kish K, Yoshikawa K, Arihara S. Limonoids and protolimonoids from the fruits of Phellodendron amurense. Phytochemistry. 1992;31(4):13351338. doi: 10.1016/0031-9422(92)80285-M

2. Ida Y, Satoh Y, Ohtsuka M, Nagasao M, Shoji J. Phenolic constituents of Phellodendron amurense bark. Phytochemistry. 1993;35(1):209-215. doi: 10.1016/S0031-9422(00)90536-3
3. Leu CH, Li CY, Yao X, Wu TS. Constituents from the Leaves of Phellodendron amurense and Their Antioxidant Activity. Chem Pharm Bull (Tokyo). 2006;54(9):1308-1311.

4. Dai LC, Si JY, Liu YL, Zhang Z. Chemical Constituents from Leaves of Phellodendron amurense Rupr. Natural Product Research and Development. 2012;24:900-902.

5. Li GZ, Li XJ, Cao L, Zhang LJ, Shen LG, Zhu J, et al. Sesquiterpene coumarins from seeds of Ferula sinkiangensis . Fitoterapia. 2015;103:222-226. doi: 10.1016/j.fitote.2015.03.022

6. Zhang LJ, Si JY, Li GZ, Li XJ, Zhang LL, Gao L, et al. Umbelliprenin and lariciresinol isolated from a long-term-used herb medicine Ferula sinkiangensis induce apoptosis and G0/G1 arresting in gastric cancer cells. RSC Advances. 2015;5(110): 91006-91017.

7. Li GZ, Li XJ, Cao L, Shen LG, Zhu J, Zhang J, et al. Steroidal esters from Ferula sinkiangensis. Fitoterapia. 2014;97:247-252. doi: 10.1016/j. fitote.2014.06.016

8. Lia HB, Yub Y, Wang ZZ, Daib Y, Gaob H, Xiaoa W, et al. Iridoid and bisiridoid glucosides from the fruit of Gardenia jasminoides. Fitoterapia. 2013;88 (7):7-11. doi: 10.1016/j.fitote.2013.03.025

9. Coates RM, Melvin LS. Total synthesis of $( \pm)$-marmin and related coumarin monoterpenes. Tetrahedron. 1970;26(24):5699-5706. doi: 10.1016/0040-4020(70)80006-0

10. Halim, AF, Saad HE, Lahloub MF, Ahmed AF. Pituranthoside from Pituranthostriradiatus. Phytochemistry.1995;40(3):927-929.

11.Li GZ, Wang JC, Li XJ, Cao L, Lv N, et al. Two new sesquiterpene coumarins from the seeds of Ferula sinkiangensis. Phytochemistry Letters. 2015;13:123-126. 